RELATO DE CASO

\title{
Tuberculose Intestinal como Causa de Obstrução Intestinal: Relato de Caso e Revisão de Literatura
}

\author{
Intestinal Tuberculosis Cause of Intestinal Obstruction: \\ Case Report and Literature Review
}

\author{
WILKER BENEDETIMENDES ${ }^{1}$; CARLOS AUGUSTO MARQUES BATISTA²; HÉLIOALVES DE LIMA²; \\ GERSON FRANÇALEITE ${ }^{3}$; JOAQUIM FERREIRADE PAULA ${ }^{4}$; WILLIAN BORBAPORTO ${ }^{1}$; MONIKAPEREIRA KIM $^{5}$; \\ CARLOS MAGNO JR ${ }^{5}$
}

\begin{abstract}
${ }^{1}$ Residente (R2) do Serviço de Cirurgia Geral do Hospital Escola Luiz Gioseffi Jannuzzi, ${ }^{2}$ Professor Titular de Clínica Cirúrgica da Faculdade de Medicina de Valença - RJ. TCBC-RJ. Mestre em Ciências; ${ }^{3}$ Professor Assistente de Clínica Cirúrgica da Faculdade de Medicina de Valença - RJ. Especialista em Proctologia e Membro Titular da Sociedade Brasileira de Coloproctologia; ${ }^{4}$ Professor Adjunto de Clínica Cirúrgica da Faculdade de Medicina de Valença - RJ. TCBC-RJ. Mestre em Ciências; ${ }^{5}$ Residente (R1) do Serviço de Cirurgia Geral do Hospital Escola Luiz Gioseffi Jannuzzi.
\end{abstract}

SOUZA HFS, SOBRAL HAC, TAGLIETTI EM, MONTEIRO EP, GAMA MRVS, FORMIGA GJS. Tuberculose Intestinal como Causa de Obstrução Intestinal: Relato de Caso e Revisão de Literatura. Rev bras Coloproct, 2009;29(4): 489-492.

RESUMO: A tuberculose intestinal geralmente é uma complicação da doença pulmonar, pela deglutição de escarro infectado. Com o advento da SIDA, houve aumento importante na incidência de tuberculose de uma forma geral. Ocorre com maior frequiência em países tropicais e subdesenvolvidos. A região ileocecal constitui o sítio de maior comprometimento. Palidez, perda ponderal, sudorese noturna e febre são os achados mais comuns ao exame físico. Dor abdominal é o principal achado no exame abdominal. A complicação mais freqüente é a obstrução intestinal. $O$ diagnóstico pré-operatório é muito difícil, sendo o de certeza dado pelo exame histopatológico ou em culturas de tecidos. $O$ tratamento cirúrgico está indicado nos casos das raras complicações.

Descritores: Tuberculose Pulmonar, Tuberculose Intestinal, Obstrução Intestinal, Válvula Ileocecal, SIDA.

\section{INTRODUÇÃO}

As primeiras descrições sobre a relação entre as formas pulmonares e extrapulmonares da tuberculose foram feitas por Laennec, em 1804. No início do século XX, era a principal causa de estenose e obstrução intestinal ${ }^{1}$. Depois de uma importante diminuição de sua incidência a partir da década de 40, a nível mundial, se notou, na década de 80 , um incremento paulatino desta enfermidade, dentre outras causas, pela epidemia do vírus HIV ${ }^{2,3}$. Estima-se que metade da população mundial esteja infectada e a cada ano pelo menos 8 milhões de novos casos são diagnosticados. No Brasil a prevalência deve atingir os 50 milhões ${ }^{4}$. Ocorre com maior freqüência em países tropicais e subdesenvolvidos, mas há relatos de aumento do número de casos inclusive nos países desenvolvidos ${ }^{2,5,6}$. A tuberculose intestinal geralmente é uma complicação da doença pulmonar ${ }^{5}$. A obstrução intestinal é uma complicação rara, mas comum no curso da tuberculose intestinal, com ou sem tratamento ${ }^{7}$. O diagnóstico pré-operatório é muito difícil, seja pela baixa incidência da doença no trato gastrintestinal, como pela simulação de diversas enfermidades abdominais, notadamente a doença de Crohn ${ }^{1,3,5,8}$. Deste modo, deve estar presente esta possibilidade, pois sem um elevado grau de suspeição, o diagnóstico é difícil de estabelecer, resultando num aumento substancial da morbimortalidade ${ }^{3,6,8}$. 
Este artigo tem como objetivo relatar um caso de obstrução intestinal por tuberculose intestinal, operado pelo Serviço de Cirurgia Geral em Valença-RJ, e fazer uma revisão da literatura sobre o tema.

\section{RELATO DO CASO}

G.A., 36 anos, masculino, branco, desempregado, natural do Paraná, residente na cidade do Rio de Janeiro, foi internado no Serviço de Clínica Médica, com quadro de dor abdominal difusa, maior em região epigástrica, iniciada há cerca de $48 \mathrm{~h}$, associado a náuseas e vômitos, hiporexia e diminuição da eliminação de fezes e flatos. Portador de SIDA e hepatite C, referia tratamento incompleto de tuberculose pulmonar há cinco anos e história de duas laparotomias exploradoras, uma devido a ferimento por arma branca e outra por projétil de arma de fogo, não sabendo informar quais lesões e procedimentos realizados. História pessoal e social de tabagismo, etilismo e uso de drogas ilícitas.

Ao exame físico, encontrava-se lúcido e orientado no tempo e espaço, corado, hipohidratado, acianótico, anictérico, afebril, e emagrecido. Aparelhos respiratório e cardiovascular sem alterações. Abdome com cicatriz cirúrgica mediana supra e infraumbilical, algo distendido, porém depressível, hipertimpânico, peristalse presente, doloroso à palpação profunda difusamente, sem sinais de peritonite. Os exames laboratoriais demonstraram apenas anemia discreta (hemoglobina $12,9 \mathrm{~g} / \mathrm{dl}$ ) e a rotina radiológica de abdome agudo uma distensão difusa do intestino delgado. Recebeu alta hospitalar após melhora clínica.

Dois dias após a alta hospitalar, foi reinternado, no Serviço de Cirurgia Geral, apresentando quadro de dor e distensão abdominal, diarréia e vômitos. Realizou TC abdominal e pélvica que mostrou marcada distensão líquida gasosa difusa das alças intestinais de delgado, até a região de íleo terminal, que exibia espessamento parietal concêntrico, associado à obliteração da gordura adjacente; observava-se ainda área de invaginação entero-entérica na altura do flanco direito. Durante internação, evoluiu com picos febris baixos e vespertinos, distensão abdominal, vômitos fecalóides e parada de eliminação de fezes e flatos, os quais foram tratados e resolvidos com medidas conservadoras, dentre elas, dieta zero, sonda nasogástrica, hidratação e analgesia parenteral, recebendo alta hospitalar.

O paciente foi readmitido, dois dias após, com o mesmo quadro acima descrito. Foi, então, submetido à laparotomia exploradora mediana supra e infraumbilical, com as hipóteses diagnósticas principais de obstrução intestinal por bridas ou invaginação intestinal, sendo encontrado os seguintes achados operatórios: distensão de alças do intestino delgado; tumoração de aspecto granulomatoso, em segmento íleo-cecal, a qual obstruía a luz intestinal e infiltrava peritoneo parietal adjacente; vários gânglios mesentéricos, também de aspecto granulomatoso. Devido ao estado geral do paciente e a extensão da lesão, foi realizado uma íleotransversoanastomose látero-lateral e biópsia excisional de um linfonodo mesentérico. O paciente evoluiu sem intercorrências e com resolução do quadro, recebendo alta hospitalar para acompanhamento ambulatorial nove dias após. O laudo histopatológico do linfonodo mesentérico caracterizou-o como granuloma tuberculóide, onde se observavam áreas de necrose de caseificação (Figuras 1 e 2), confirmando, assim, um caso de tuberculose intestinal.

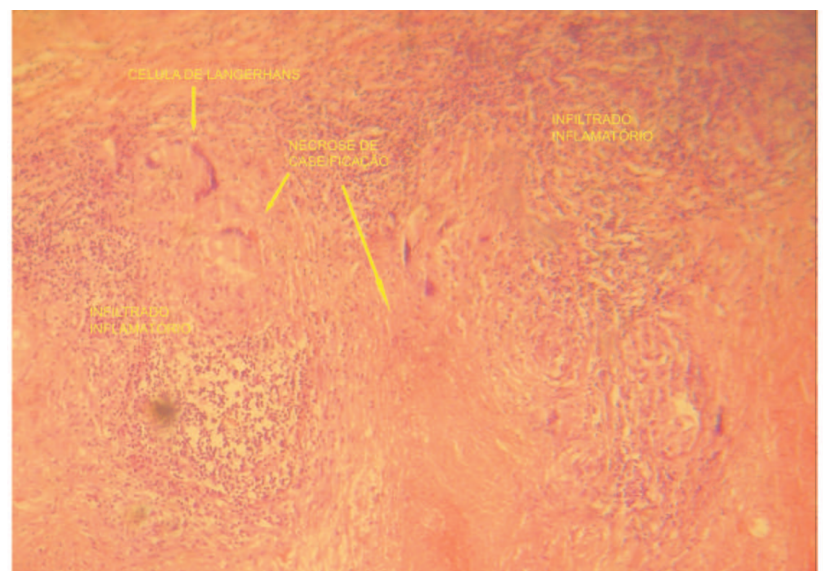

Figura 1 - Aspecto microscópico do granuloma tuberculóide: lâmina em coloração HE, aumento100x.

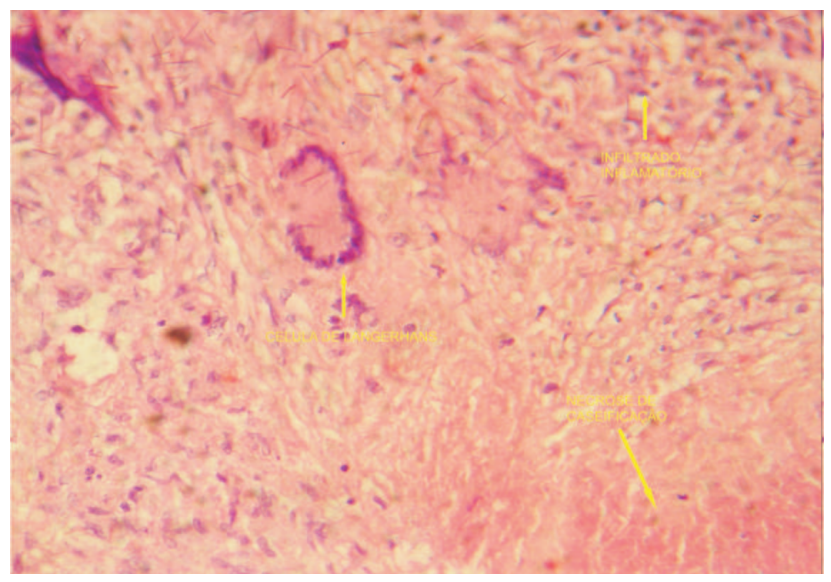

Figura 2 - Aspecto microscópico do granuloma tuberculóide: lâmina em coloração HE, aumento $250 x$. 
O paciente foi acompanhado ambulatorialmente pelo Serviço de Cirurgia Geral e pela DIP, sendo instituído o tratamento farmacoterápico para tuberculose.

\section{DISCUSSÃO}

Com a epidemia pelo HIV, houve um aumento da incidência da tuberculose pulmonar, acompanhado por um aumento da incidência de manifestações extrapulmonares que chegam a atingir os $50 \%{ }^{3}$. Pacientes portadores de SIDA são particularmente susceptíveis à tuberculose extrapulmonar, com incidência de até $76 \%$ em algumas séries, sendo mais afetados os sistemas linfático, urinário, digestivo e nervoso cen$\operatorname{tral}^{4,5,9,10}$.

A tuberculose intestinal geralmente é uma complicação da doença pulmonar, pela deglutição de escarro infectado; pode surgir, de forma isolada, pela ingestão de leite contaminado (embora rara atualmente), por disseminação hematogênica e ainda por implante de bacilos de focos contíguos ${ }^{1,5,6,9,11,12,13}$.

Ocorre com maior frequiência em países tropicais e subdesenvolvidos, como Índia e Nigéria, com relato de aumento do número de casos em diversos outros países ${ }^{2,5,10,12,14}$. Na Índia, cerca de 7\% das internações por obstrução ou perfuração intestinal são causados por tuberculose ${ }^{3,5,7,10}$. A idade média de ocorrência, em diversos estudos, foi de 46 anos. Acomete mais os homens que as mulheres, numa proporção de $3: 1^{5,15,16}$.

A estase fisiológica do íleo e região ileocecal e a alta concentração de tecido linfóide nesta área têm sido apontados como principais fatores pela qual a região ileocecal constituiu o sítio de maior comprometimento, em cerca de $85 \%$ dos $\operatorname{casos}^{2,3,4,6,7,10,11,12,13,14}$. Excepcionalmente, são registradas úlceras na língua, lábios e faringe; esôfago e estômago são ainda mais $\operatorname{raros}^{4,11,12,13}$.

Em termos de anatomopatologia, há três ou quatro tipos de lesões, segundo diversos autores: hipertrófica, ulcerativa, hipertrófica-ulcerativa e esclerótica ${ }^{3,4,5,12,14,15}$. A forma hipertrófica é a causa mais comum de obstrução intestinal ${ }^{7}$.

Palidez, perda ponderal, sudorese noturna e febre são os achados mais comuns ao exame físico. $\mathrm{O}$ principal achado no exame abdominal é a dor, variando de $85 \%$ a $100 \%$ dos casos, sendo, às vezes, acompanhado de massa palpável, diarréia e perda ponderal $1^{2,3,4,7,8,10,11,12,13,15,16}$. A complicação mais fre- qüente é a obstrução intestinal, na maioria das vezes intermitente e parcial ${ }^{3,5,7}$. Perfuração e hemorragia intestinais também podem ocorrer, embora raras. Fístulas anais são muito raras ${ }^{3,4,5,15}$.

O diagnóstico pré-operatório é muito difícil e $\operatorname{raro}^{3,5,7,10,14}$. O diagnóstico específico só poderá ser assegurado pela demonstração histológica de bacilos álcool-ácido resistentes na lesão ou pela cultura, presença de necrose caseosa e evidências histológicas específicas em linfonodos regionais ${ }^{3,67,7,8,10,12,13}$. Uma anamnese bem conduzida e um exame físico meticuloso podem levantar suspeita. Anemia discreta pode estar presente ${ }^{2,10,12,16}$. O PPD é positivo $50 \%$ a $70 \%$ dos casos, mesmo tendo valor diagnóstico limitado, pois não distingue entre doença ativa e sensibilização prévia $^{1,5,10,12,14,16}$. A radiografia simples de tórax pode mostrar alterações de tuberculose pulmonar em $20 \%$ a $50 \%$ dos $\operatorname{casos}^{2,3,6,10,14,16}$. As lesões intestinais são encontradas associadas à forma pulmonar em $70 \%$ a 87\%; nos pacientes com a manifestação hipertrófica havia relato de tuberculose tratada há anos ${ }^{15}$. A radiografia simples de abdome mostra distensão de intestino delgado com níveis hidroaéreos em 50\% dos casos; nos exames baritados, evidenciam-se alças espessadas, espásticas, de contornos irregulares e ulcerações superficiais $^{5,10}$. A obstrução crônica leva ao aparecimento de segmentos de intestino com enormes dilatações ${ }^{12}$. A TC de abdome pode mostrar desde leve espessamento da parede do ceco e íleo terminal até o aparecimento de massa de linfonodos englobando a região ileocecal ${ }^{5,8,16}$. A colonoscopia com biópsia pode ser definidora, desde que identifique a lesão, para o diagnóstico em $80 \%$ dos $\operatorname{casos}^{2,3,4,8,16}$.

O diagnóstico diferencial deve ser feito principalmente com a doença de Crohn e com tumores malignos do cólon ${ }^{3,4,5,8}$. A linfadenopatia pode dar a impressão de linfoma ${ }^{4}$. Retocolite ulcerativa inespecífica, sarcoidose, amebíase, histoplasmose, apendicite, entre outras também devem ser consideradas ${ }^{1,6}$.

Os dados da literatura mostram que $90 \%$ dos casos de tuberculose gastrointestinal respondem favoravelmente à terapêutica médica, desde que iniciada precocemente ${ }^{2,3,4,6}$. A terapia cirúrgica está indicada nos casos das raras complicações como perfuração livre, perfuração com abscesso, obstrução, fístulas ou hemorragias, ou quando não está definido o diagnósti$\mathrm{co}^{2,3,4,6,10,12,14,15}$. O quadro mais comum que resulta em indicação cirúrgica é a obstrução intestinal, relacionado à forma hipertrófica que responde mal a 
farmacoterapia, muitas vezes constituindo em laparotomias complexas, com a realização de ressecções extensas de delgado com cólon ascenden$\mathrm{te}^{5,10,15}$. A hemicolectomia direita provou, para muitos autores, ser mais curativa e efetiva para lesões envolvendo a região íleo-cecal ${ }^{7}$. Dificuldades são encontradas por aderências firmes entre alças, grande exten- são de intestino envolvido e pelo estado geral comprometido do paciente, sendo uma derivação ileocólica um procedimento mais seguro ${ }^{5,7,14}$. No presente caso, foi indicado a ileotransversoanastomose devido à extensão da lesão e o precário estado geral do paciente. $\mathrm{O}$ tratamento poliquimioterápico deve ser instaurado no seguimento pós-cirúgico ${ }^{2,14}$.

\begin{abstract}
Intestinal tuberculosis occurs often as a pulmonary disease complication, when infected sputum is swallowed. With AIDS there was a significant increasement of tuberculosis in all of it forms. It happens more often in tropical and third world countries. The ileocecal is the site with most commitment. Paleness, weight loss, night sweats and fever are the most common findings on physical examination. Abdominal pain is the main finding in the abdominal examination. The most frequent complication is intestinal obstruction. The preoperative diagnosis is very difficult, and the certainty provided by histopathological examination or in tissue culture. Surgical treatment is indicated in cases of rare complications.
\end{abstract}

Keywords: Pulmonary Tuberculosis, Intestinal Tuberculosis, Intestinal Obstruction, Ileocecal Valve, AIDS.

\section{REFERÊNCIAS}

1. Rubio T, Gaztelu MT, Calvo A, Repiso M, Sarasíbar H, Bermejo FJ, et al. - Tuberculosis abdominal. Anales Sis San Navarra 2005; 28(2): p 257-260.

2. Nari GA, Dalale J, Ponce OH, Yanucci V - Obstrucción intestinal por tuberculosis colónica. Presentación de dos casos. Rev Esp Enferm Dig (Madrid) 2006; 98(2) p 144-150.

3. Lopes L, Certo M, Ramada J, Soares J, Guimarães J, Ribeiro M, et al. - Tuberculose intestinal. GE - J Port Gastrenterol 2004; 11: p 25-29.

4. Loureiro MP, Cruz P, Fontana A, Weigmann SC, Shibata M Tuberculose intestinal - diagnóstico e ressecção minimamente invasivos. Rev bras videocir 2006; 4(1): p 13-16.

5. Sá Ribeiro FA, Alves ALF - Doenças inflamatórias e infecciosas. In: Vieira OM, Chaves CP, Manso JEF, Eulálio JMR, editores. Clínica Cirúrgica: fundamentos teóricos e práticos. São Paulo: Atheneu; 2000. p. 253-58.

6. Barreto JBP, Carneiro Neto JD, Lima Filho PWL, Souza YLMS - Tuberculose entérica com fístula colo-cutânea espontânea: relato de caso. Rev bras Coloproct 2003; 23(2): p 108-111.

7. Nguyen VH - Intestinal obstruction due to tuberculosis. Asian J Surg 2002; 25(2): p 145-148.

8. Bromberg SH, Faroud S, Castro FF, Morrone N, Godoy AC, França LCM - Tuberculose ileocecal isolada simulando neoplasia maligna e doença de Crohn. Rev Assoc Med Bras 2001; 47(2): p 125-128.

9. Grisi SJFE, Cardoso AC, Bellizia L, Escobar AMU - Tuberculose peritoneal: relato de caso e comparação de métodos diagnósticos. Pediatria (São Paulo) 2001; 23(1): p 100-105.
10. Chuttani HK, Sarin SK - Intestinal tuberculosis. Ind J Tub 1985; 32(3): p 117-125.

11. Torres Filho SR Tuberculose. In: Tavares W, Marinho LAC, editores. Rotinas de diagnóstico e tratamento das doenças infecciosas e parasitárias. São Paulo: Atheneu; 2005. p. 102340.

12. Tovo CV, Mondin M, Schneider NC, Damo DF - Tuberculose intestinal: relato de 2 casos. Mom. \& Perspec. Saúde 2003; 16(2): p 32-35.

13. Neves JS, Dettoni VV, Pissinali CS, Peçanha PM Tuberculose. In: Neves J, editor. Diagnóstico e tratamento das doenças infectuosas e parasitárias. $2^{\mathrm{a}}$ ed. Rio de Janeiro: Guanabara Koogan; 1983. p. 496-524.

14. Townsend CM, Jr, Thompson JC Intestino delgado. In: Schwartz SI, Shives GT, Spencer FC, editores. Princípios de Cirurgia. $6^{\text {a }}$ ed. Rio de Janeiro: Interamericana/McGraw-Hill; 1996. p. 1047-81.

15. Nadal CRM, Nadal SR, Klug WA, Capelhuchnik P - Tuberculose intestinal - formas complicadas. Rev bras Coloproct 1991; 11(1): p 25-28.

16. Yriberry S, Cervera Z, Soriano C, Frisancho O, Machado A, Zumaeta E - Tuberculosis digestiva en el Hospital Nacional Edgardo Rebagliati Martins (HNERM): un estudio retrospectivo de 5 años (1993-1998). Rev gastroenterol Perú 1998; 18(3): p 238-249.

\section{Endereço para correspondência:}

Rua Comendador Antônio Jannuzzi, 183, bloco B02, apto 303

Bairro Belo Horizonte - Valença - RJ

CEP 27600000

E-mail: aindatonorio@yahoo.com.br

Telefone: (24) 9981-0360 